\title{
Reconfigurable Radar Transmitter Based on Photonic Microwave Signal Generation
}

\author{
Francesco Laghezza $^{\# 1,2}$, Fabrizio Berizzi ${ }^{2}$, Amerigo Capria ${ }^{\# 3}$ Andrea Cacciamano $^{\# 4}$ \\ Paolo Ghelfi ${ }^{\dagger 5}$, Giovanni Serafino**6 ${ }^{*}$, Antonella Bogoni ${ }^{\dagger 7}$ \\ ${ }^{\#}$ RaSS Center - CNIT, via Moruzzi 1, 56124 Pisa, Italy \\ *Dept. of Information Engineering - University of Pisa, via Caruso 16, 56122 Pisa, Italy \\ ${ }^{\dagger}$ Nat'l Lab of Photonic Networks - CNIT, via Moruzzi 1, 56124 Pisa, Italy \\ **Scuola Superiore Sant'Anna, via Moruzzi 1, 56124 Pisa, Italy \\ \{ ${ }^{1}$ rancesco.laghezza, ${ }^{2}$ f.berizzi, ${ }^{3}$ amerigo.capria, andrea.cacciamano\}@iet.unipi.it \\ $\left\{{ }^{5}\right.$ paolo.ghelfi, ${ }^{7}$ antonella.bogoni\}@cnit.it \\ 'g.serafino@sssup.it
}

\begin{abstract}
In this paper we propose a photonic technique for a reconfigurable microwave signal generation based on the beating in a photodiode of two laser modes from a regenerative Fiber Mode-Locked Laser (FMLL). The excellent performance of this kind of pulsed laser guarantees high stability to the generated microwave signal even at ultra high frequencies (up to W band). Therefore, by using the proposed architecture, the performance of a reconfigurable full digital coherent radar system can be enhanced in terms of Moving Target Indicator (MTI) improvement factor. Moreover, thanks to the achievable high repetition rates and the coherence properties of the FMLL, this laser scheme has also been proposed for digitizing the received signal by electro-optical sampling. Thus the advantage of using just one device for signal generation in both the transmitter and receiver chain, makes the proposed solution a cost effective architecture for microwave signal generation. Differently from the microwave synthesizers, whose performance strongly deteriorate with increasing frequencies, the photonic radio frequency generation always shows an excellent spectral purity. The results show excellent spectral purity above $5 \mathrm{KHz}$ for the proposed technique compared to a state of the art Agilent synthesizer even though the timing jitter increases for integration time greater than $10 \mathrm{msec}$. In order to achieve the same stability performance at both high and low frequencies a Phase Locked Loop between the laser and a synthesizer could be used.
\end{abstract}

\section{INTRODUCTION}

In the last few years the intriguing concept of very high performance reconfigurable radar transmitter is becoming feasible, mainly thanks to the wide and growing development of digital solutions for radar system applications. In this scenario a digital approach could have a lot of advantages with respect to the classical radar architecture in terms of transceiver module size and cost, instantaneous pulse bandwidth, software based capability, Multi Functional Radar systems (MFRs) and so on. In addition, the demand of a new generation of surveillance radar, satellite communications, remote sensing and Ground Penetrating Radar (GPR), as well as biomedical imaging, automotive and security systems, has driven the technology development in this direction [1]-[2]. For this new kind of radar the adaptivity of the microwave radio frequency generation according to the changing scenarios is one of the most important requirement, together with high operating frequencies, spectral purity and flexible baseband signal generation. Therefore, both high frequency oscillators with very low phase noise for up/down conversion, and direct RF analogue to digital converters are required.

In a conventional radar system the spectral purity of the microwave signal also depends on the frequency stability of a STAble Local Oscillators (STALO). In order to satisfy the microwave regime and to improve the RF signal stability, the combination of different type of oscillators (acoustic, electrical and so on) is necessary. Some of the weaknesses of this implementation is the usage of low frequency resonant modes, the necessity of a great number of multiplication stages, and the strongly frequency dependent performance of microwave signal in terms of amplitude and phase jitter [3]. This instability can induce a large phase noise on the target and clutter echo and can modify the signal spectrum, masking the presence of a small moving target echo. Moreover it can produce ambiguity on the target radial distance and Doppler frequency estimation, constraining the time delay clutter canceller in MTI processing [4].

In this paper we propose a photonic technique for realizing a reconfigurable microwave signal generation based on the beating of two laser modes from a stable regenerative Fiber Mode-Locked Laser (FMLL). Thanks to its excellent stability, high repetition rates and its potential for integration, this architecture have also been proposed as electro-optical sampler for the received signal [5]. The performance of a reconfigurable full digital coherent radar system will be enhanced in terms of MTI improvement factor. The proposed architecture, based on the use of just one device for both transmitter and receiver chain, also represents a cost effective solution for microwave signal generation. Moreover the nature of this approach guarantees the same performance up to ultra high frequencies. The results show excellent spectral purity above $5 \mathrm{KHz}$ for the proposed technique compared to a state of the art Agilent synthesizer even though the timing jitter increases for integration time greater than $10 \mathrm{msec}$. In order to achieve the same stability performance at both high and low 
frequencies a Phase Locked Loop between the laser and a synthesizer could be used.

After a brief introduction, the architecture of the reconfigurable radar transmitter system is reported in section II, then a first photonic RF generation experiment at $\mathrm{X}$ band will be presented in section III where the performance in terms of time and amplitude jitter will be compared with other microwave signal generation techniques. The main results will be then summarized in section IV.

\section{RECONFIGURABLE MicROWAVE RADAR TRANSMITTER}

The logical architecture of the reconfigurable full digital radar transmitter is shown in Fig. 1, where electrical and optical devices are highlighted in light blue and yellow respectively.

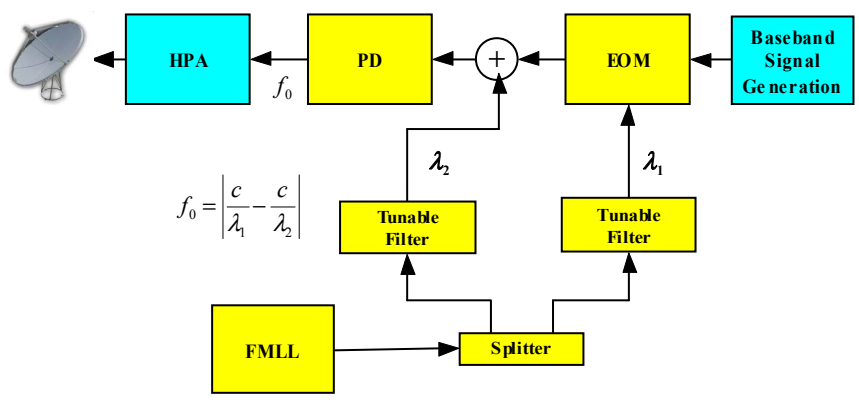

Fig. 1 Reconfigurable microwave radar transmitter architecture.

The generation of the RF carrier frequency is realized by the beating in a photodiode (PD) of two laser modes from a fiber mode locked laser. The FMLL is a fiber laser with excellent stability thanks to the auto regenerative feedback. It is able to produce narrow optical pulses with repetition rate in the range from $1 \mathrm{GHz}$ up to $40 \mathrm{GHz}$. The optical spectrum is composed of a number of modes, whose spacing is equal to the pulse repetition rate. All the laser modes are phase locked with each other thanks to the mode locking condition and the regenerative feedback that keeps the modes well locked [6].

The selection of the two beating modes is realized by splitting the optical signal emitted by the FMLL in two arms by an optical splitter with a 50\% splitting ratio. Each replica of the signal is filtered by an optical tuneable filter in order to select only one mode from the spectrum. Along the two arms two different modes are filtered, at a frequency detuning that is a multiple of the laser repetition rate. The integration of splitter and tuneable filters is needed in order to avoid interference noise on the beating signal.

The radio frequency up conversion system can be realized by an electro-optical modulator (EOM) such as a Mach Zehnder Interferometric Modulator (MZIM) biased at its quadrature point [8], inserted in one of two arms. The EOM is driven by a baseband signal generation block which addresses the digital synthesis of arbitrary waveforms - such as pulse trains, coded waveforms, frequency modulated waveforms like chirp - with an adaptive bandwidth optimized according to the targets of interest and the environment. For example some of the key features of the baseband signal generation are the frequency, phase and amplitude sweeping capability, the number of bits of the Digital to Analogue Convert (DAC) and so on.

The two modes, the modulated and the unmodulated ones, are then combined together in a coupler, and sent in a photodiode which realizes the heterodyning, generating the final microwave signal. Finally, the High Power Amplifier (HPA) provides the necessary power for transmission [9].

This architecture has the capability for reconfigurable transmission. In fact, by tuning the filters it is possible to select laser modes at different frequency detuning, thus generating in the photodiode a RF carrier at any multiple frequency of the laser repetition rate. Moreover the use of a digital baseband signal generator allows to match the RF signal modulation to the target and environment.

\section{RADIO FREQUENCY SIGNAL GENERATION EXPERIMENT}

The architecture set up of the $\mathrm{X}$ band experiment is shown in Fig. 2, where the FMLL provided by PhoTrix is operating at $10 \mathrm{GHz}$ and it is able to emit 4 picoseconds pulses centred at $1550 \mathrm{~nm}$.

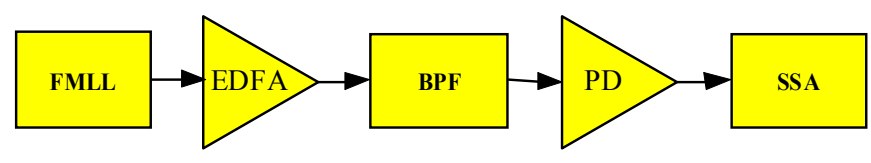

Fig. 2 Experiment architecture.

An Erbium-Doped Fiber Amplifier (EDFA) is used before the filtering process to guarantee sufficient optical power at the photodiode. A Band Pass Filter (BPF) with a $3 \mathrm{~dB}$ bandwidth of $0.15 \mathrm{~nm}$ and steep edges has been used to select two adjacent laser lines. Fig. 3 shows the filtered optical spectrum taken by an optical spectrum analyzer with a resolution bandwidth of $0.01 \mathrm{~nm}$ (corresponding to 1.25 $\mathrm{GHz}$ ), compared with the original spectrum of the FMLL. The undesired modes are suppressed more than $20 \mathrm{~dB}$.

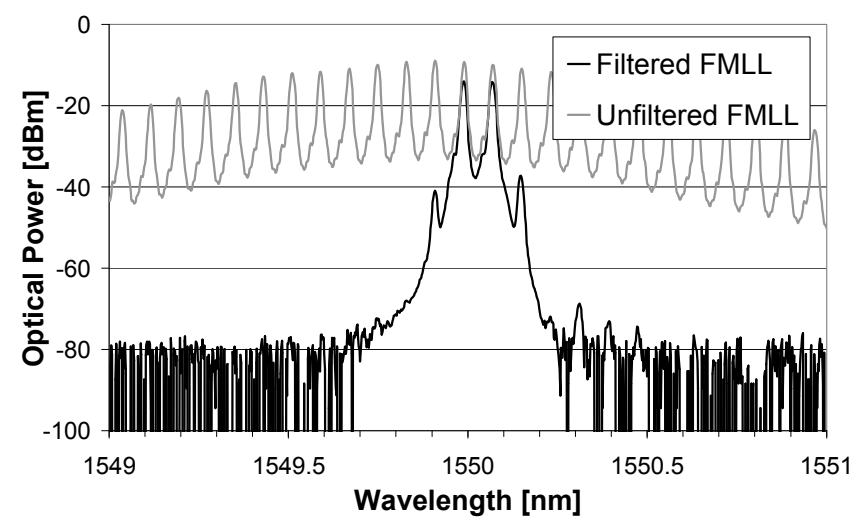

Fig 3 . Optical spectrum of the FMLL and the two selected modes. Trace acquired with a resolution bandwidth of $0.01 \mathrm{~nm}$ (about $1.25 \mathrm{GHz}$ ) 
The two selected modes are then injected in an amplified photo-detector with a 3dB-bandwidth of $11 \mathrm{GHz}$. Since the photodiode realizes a quadratic function of its optical input, the two inserted laser modes beat together generating a RF signal at a frequency that is equal to their frequency detuning, i.e. at $10 \mathrm{GHz}$.

The microwave signal thus generated is analyzed by means of a Signal Source Analyzer (Agilent E5052A) [6]. The obtained result is shown in Fig. 4, where the phase noise of the beating signal is compared with other RF sources, namely a VCO at $10 \mathrm{GHz}$ (Narda FFCM), a state of the art synthesizer (Agilent E8257D), and the heterodyning of two DistributedFeedback (DFB) lasers with a frequency detuning of $10 \mathrm{GHz}$ (EXFO IQS-2400 modules).

The comparison of Fig. 4 is realized for a frequency offset from the RF carrier in the range from $100 \mathrm{~Hz}$ to $40 \mathrm{MHz}$. These range limits are due respectively to the usual time duration of the radar measurements (generally longer than 1 $\mathrm{ms}$ ), and to the intrinsic limits of the measuring instruments.

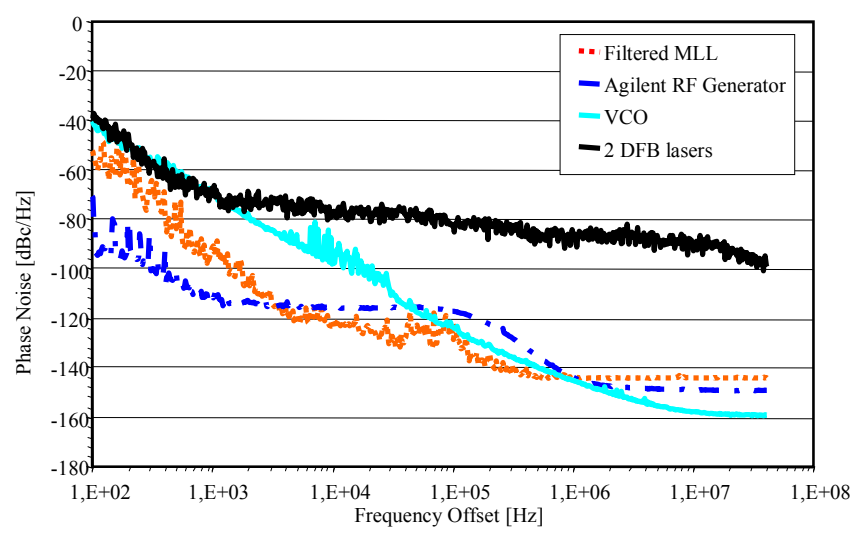

Fig. 4 Comparison of phase noise levels for different radio frequency sources at $10 \mathrm{GHz}$.

As can be seen from the graph in Fig. 4, the proposed architecture (orange line) and the state of the art RF synthesizer provided by Agilent (blue line) show comparable behaviours, while the other RF generation methods present much worse performance, with phase noise levels tens of $\mathrm{dB}$ higher in large part of the analysis range. The comparison between the proposed scheme and the state of the art synthesizer is reported in more details in Fig. 5. In the graph the analysis has been extended to an observation time as long as $1 \mathrm{~s}$ to take into consideration the most requiring radar applications, as for example tracking or imaging, when the phase coherence must be maintained for the whole observation time necessary to obtain an electromagnetic image. Fig 5 shows that the proposed RF generation technique performs better than the synthesizer in a wide range of frequency, from about $5 \mathrm{KHz}$ to $1 \mathrm{MHz}$. For offset frequencies above $1 \mathrm{MHz}$, even though the synthesizer shows less phase noise, both the RF sources performs well. On the other hand, for offset frequencies lower than $5 \mathrm{KHz}$, the state of the art synthesizer shows extremely low phase noise, while our proposed photonic RF generation becomes more instable, although it's behaviour is still better than other RF sources (see Fig. 4).

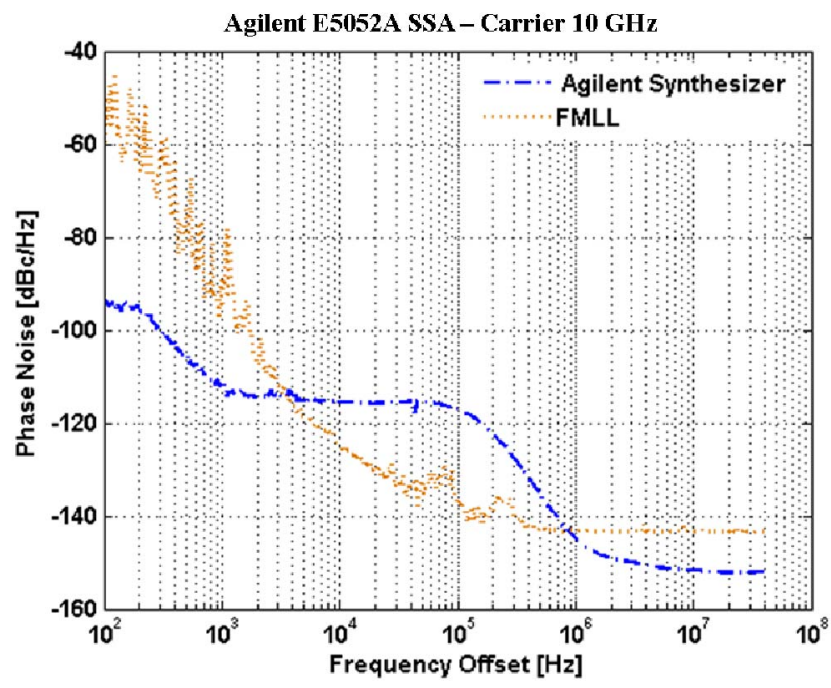

Fig. 5 Phase noise level for filtered FMLL (orange) and for Agilent E8257D synthesizer (blue).

In a Fig. 6 the electrical spectra analysis of this two sources has been presented. Both the FMLL and the microwave synthesizer show the same signal to noise floor ratio higher than $80 \mathrm{~dB}$ and present very narrow linewidths.

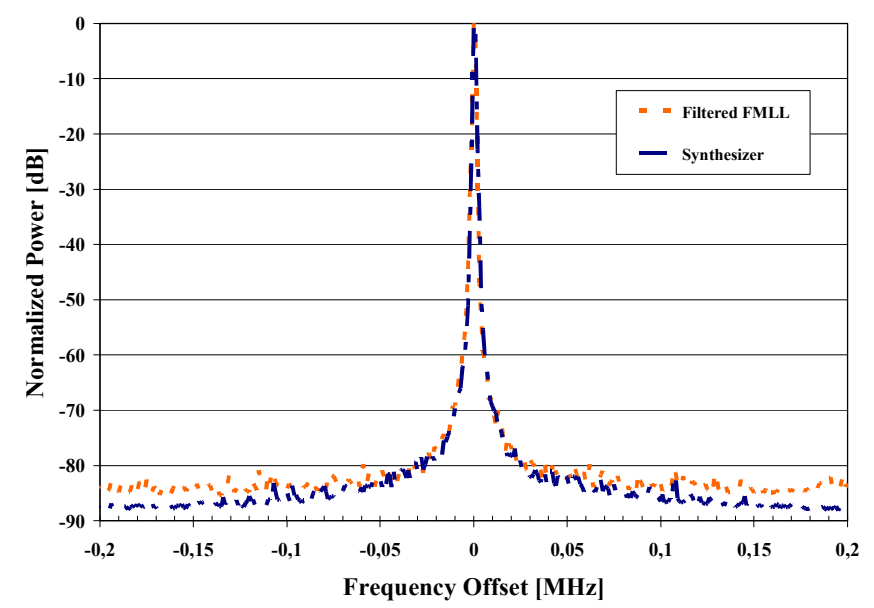

Fig. 6 Electrical spectra of different radio frequency sources with a resolution bandwidth of $100 \mathrm{~Hz}$.

Integrating the phase noise curve reported in Fig. 5 it is possible to calculate the timing jitter of the RF signal. The integration interval should be chosen accordingly with the observation time. We performed the calculation for different frequency intervals. The results comparing the proposed RF generation method and the state of the art synthesizer are 
reported in Table I. When the phase noise is integrated between $1 \mathrm{KHz}-40 \mathrm{MHz}$, the obtained jitter is $18.792 \mathrm{fsec}$ for the filtered MLL and 15.232 fsec for the Agilent synthesizer, whereas when the integration time starts from $1 \mathrm{sec}$ the timing jitter from the photonic architecture is larger than the microwave state of the art source. In Table 1 the jitter performance for different limits of integration are summarized. For example when the frequency interval ranging from $5 \mathrm{KHz}$ to $40 \mathrm{MHz}$ the proposed scheme present a time jitter of about $15 \mathrm{fsec}$ while the jitter of Agilent synthesizer is 16 fsec.

TABLE I

TIMING JITTER OF TWO DIFFERENT MICROWAVE SOURCES

\begin{tabular}{|c|c|c|}
\hline $\begin{array}{c}\text { Frequency } \\
\text { Interval }\end{array}$ & $\begin{array}{c}\text { Filtered } \\
\text { FMLL } \\
\text { architecture }\end{array}$ & $\begin{array}{c}\text { Agilent } \\
\text { E8257D } \\
\text { synthesizer }\end{array}$ \\
\hline $1 \mathrm{~Hz}-40 \mathrm{MHz}$ & $108.156 \mathrm{psec}$ & $149.713 \mathrm{fsec}$ \\
\hline $5 \mathrm{~Hz}-40 \mathrm{MHz}$ & $4.06 \mathrm{psec}$ & $28.168 \mathrm{fsec}$ \\
\hline $100 \mathrm{~Hz}-40 \mathrm{MHz}$ & $508.522 \mathrm{fsec}$ & $16.22 \mathrm{fesc}$ \\
\hline $1 \mathrm{KHz}-40 \mathrm{MHz}$ & $18.792 \mathrm{fsec}$ & $15.232 \mathrm{fsec}$ \\
\hline $5 \mathrm{KHz}-40 \mathrm{MHz}$ & $15 \mathrm{fsec}$ & $16 \mathrm{fsec}$ \\
\hline
\end{tabular}

An amplitude noise upper bound lower than $0.1 \%$ has been evaluated for the used sources by integrating the electrical spectra between $1 \mathrm{KHz}$ and $40 \mathrm{MHz}$ [10], limited by the resolution bandwidth of the spectrum analyzer.

It should be noted that in order to achieve the same stability performance at both high and low frequencies it is possible to lock the laser to a synthesizer by a Phase Locked Loop (PLL) [10]. In this case the total instability is derived form the laser at high frequency and from the microwave source at low frequency. It is worth underlining that, while the performance of the synthesizer strongly degrades when the frequency increases, the stability characteristics of the proposed reconfigurable technique remains stable even for high repetition rates. The only drawback in this configuration is a little phase noise enhancement due to the selection of two non adjacent laser lines by the tuneable filters.

\section{CONCLUSIONS}

The architecture of a photonic microwave radar transmitter based on the beating in a photodiode of two laser modes from a FMLL has been presented in this paper and the stability performance in terms of amplitude and time jitter of the radio frequency source at $10 \mathrm{GHz}$ has been evaluated. The results show excellent spectral purity above $5 \mathrm{KHz}$ for the proposed technique compared to a state of the art Agilent synthesizer even though the timing jitter increases for integration time greater than $10 \mathrm{msec}$. In order to achieve the same stability performance at both high and low frequencies a Phase Locked Loop between the laser and a synthesizer could be used.

In addition, with the proposed architecture the evaluated performance are guaranteed up to ultra high frequencies differently from conventional microwave sources and it represent a cost effective solution for microwave signal generation. Finally this architecture has the capability for reconfigurable transmission in fact, by tuning the filters it is possible to select laser modes at different frequency detuning, thus generating in the photodiode a RF carrier at any multiple frequency of the laser repetition rate.

\section{REFERENCES}

[1] C. J. Peacock, G. S. Pearson, "Digital Radar," Proc. of the IET International Conference on Radar System, 2007.

[2] Y. K. Chan, S. Y. Lim, "Synthetic Aperture Radar (SAR) Signal Generation," Progress in Electromagnetics Research B, Vol. 1, pp 269-290, 2008

[3] M. Dispenza, A. Secchi, L. Pierno, A. Fiorello, G. Parca, R. Capolupo, S. Betti, "Tuneable Optoelectronic Oscillator Based on a Frequency Shifter ," Proc. of the 6th European Radar Conference, 2009, paper 262.

[4] Sung-Chan Song, Yun-Seok Hong, "A New Approach for Evaluating the Phase Noise Requirements of STALO in a Doppler Radar," Proc. of the 37th European Microwave Conference, October 2007, Munich, Germany.

[5] L. Pierno, M. Dispenza, G. Tonelli, A. Bogoni, P. Ghelfi, L. Potì, "A Photonic ADC for radar and EW applications based," MWP 2008, Australia.

[6] P. Ghelfi, G. Serafino, F. Berizzi, A. Bogoni, "Generation of Highly Stable Microwave Signals Based on Regenerative Fiber Mode Locking Laser “, submitted to Laser and Electro-Optics Conference, CLEO 2010

[7] L. Goldberg, H. F. Taylor, J. F. Weller, D. M. Bloom, "Microwave Signal Generation with injection laser diodes," Electron. Lett., vol. 19, no. 13, pp. 491-493,Jun. 1983.

[8] Charles H. Cox III, "Limits on the performance of RF-Over Fiber Links and Their Impact on Device Design," IEEE Transactions on Microwave Theory and Techniques, vol. 54, no. 2, February 2006.

[9] A. Bogoni, F. Berizzi, "PHOtonic-based full Digital Radar," Nat'1 Lab of Photonic Network and RaSS Center, Tech. Rep. WP1-Feasibility Study, 2009.

[10] L. Banchi, F. Rossi, M. Ferianis, A. Bogoni, L. Potì, P. Ghelfi, "Synchronization of $3 \mathrm{GHz}$ repetition rate harmonically mode-locked fiber laser for optical timing applications," Proceedings of DIPAC 2007 the 37th European Microwave Conference, October 2007, Italy. 